\title{
Research Report: Implicit and Explicit Measures of Sexism Predicting Men's Interviewing Behaviors
}

\author{
J. T. Nadler \\ Southern Illinois University Edwardsville \\ E. Voyles \\ Southern Illinois University Edwardsville \\ V. Brooks \\ Southern Illinois University Edwardsville \\ M. VanCleave \\ Southern Illinois University Edwardsville
}

\begin{abstract}
Implicit (Indirect measures of bias often capturing unconscious biases such as eye tracking) and explicit (direct survey measures capturing conscious biases such as the Ambivalent Sexism Inventory (ASI): Glick and Fiske, 2001) sexism were measured in men who then interviewed a female job seeker. The results of this study examined explicit outcomes of the interviews (ratings) as well as implicit outcomes (non-verbal behaviors). Higher levels of implicit gender bias (Eye tracking) was significantly related to the outcomes and was a better predictor of sexist non-verbal behaviors than the explicit measure.
\end{abstract}

Keywords: sexual harassment, implicit measures, interview evaluations

\section{INTRODUCTION}

\section{Eye Tracking, Sexism, and Gender Bias}

Eye tracking is emerging as a versatile tool for inferring biases implicitly (Duchowski, 2002). For example, Gerpott, Lehmann-Willenbrock, Sivlis, and Van Vugt's (2017) found eye-tracking results could be used to identify emergent leadership behaviors. Past eye-tracking studies have shown that sexist men's initial visual fixations focus on women's secondary sexual traits (breasts) more than any other region when viewing images of women (Hewig et al., 2008; Suschinsky et al., 2007). Additionally, in attractiveness fixation studies where facial details were blurred, both men and women fixated more on the chest than any other region (Cornelissen et al., 2009). Finally, several non-eye tracking studies have analyzed correlations between attending to the physical attractiveness of women and men's sexist beliefs in hiring and promotion decisions (Forbes, Collinsworth, Jobe, Braun, \& Wise, 2007; Swami \& Tovée, 2013; Zelazniewicz \& Pawlowski, 2011). Hayes, Pham, Phillips, and Nadler (2019) found significant correlations with eye 
tracking fixations when viewing images of professional women and other measures of sexism. This work suggests eye tracking may serve as a useful implicit measure of gender bias.

Overt bias targeting women directly violates U.S. law and has become much less culturally acceptable (Nadler \& Stockdale, 2012). However; evidence continues to support gendered differences in work outcomes favoring men over women from the smallest micro-interactions to overall trends in career progression across the United States (U.S; Stockdale \& Nadler, 2013). Thus, more recent research has focused on less overt mechanisms of bias such as micro-aggressions and non-verbal behaviors (Gartner, \& Sterzing, 2016; Nadler \& Stockdale 2012).

Our study utilized explicit and implicit measures of sexism (gender bias favoring men over women in organizational decisions to examine potential organizational outcomes in an employment interview setting. The outcomes included an explicit outcome (interview ratings) and an implicit outcome (non-verbal behaviors directed at the interviewee). Based on previous research of implicit and explicit sexism the following hypotheses were tested.

Hypothesis 1: The implicit bias measure will be positively correlated with the explicit measures of sexism.

Hypothesis 2: The measure of implicit bias will be more predictive of subsequent explicit applicant ratings and implicit non-verbal behaviors.

Hypothesis 3: The measure of implicit bias will be more predictive of subsequent explicit applicant ratings than the measure of explicit bias.

\section{METHODS}

\section{Explicit Measure of Gender Bias}

Participants at a mid-Western state university (men, average age of 20 , primarily White $(92 \%)$ and all self-reporting heterosexual) were given the ASI (22 Likert scale items, Cronbach's Alpha 0.78; Glick \& Fiske, 1996) as part of a mass pre-test (Session $1 ; N=223$ ). For the purposes of this study the two subscales of the ASI (benevolent and hostile sexism sub-scales) were used as the explicit measure of gender bias.

\section{Implicit Measure of Gender Bias}

Of the 223 men in Session 1, 66 men were asked to Session 2 where they completed an eye tracking task rating (they indicated based on looks alone if they would ask this person for an interview; this data was not used for this study) a series of 12 women job applicants' pictures cropped to present face and upper torso always presented in the same area of the screen. During each image presentation ( 5 seconds) the participants' gaze was tracked specifically examining how much time each participant focused on the torso (chests) of the women presented. Eye tracking was completed using ASL's Eye-Trac 6 Desktop. For an example of typical 'heat maps' of two participants 5 seconds of gaze, see Figure 1. 


\section{FIGURE 1 \\ EXAMPLE OF EYE TRACKING FIXATIONS}

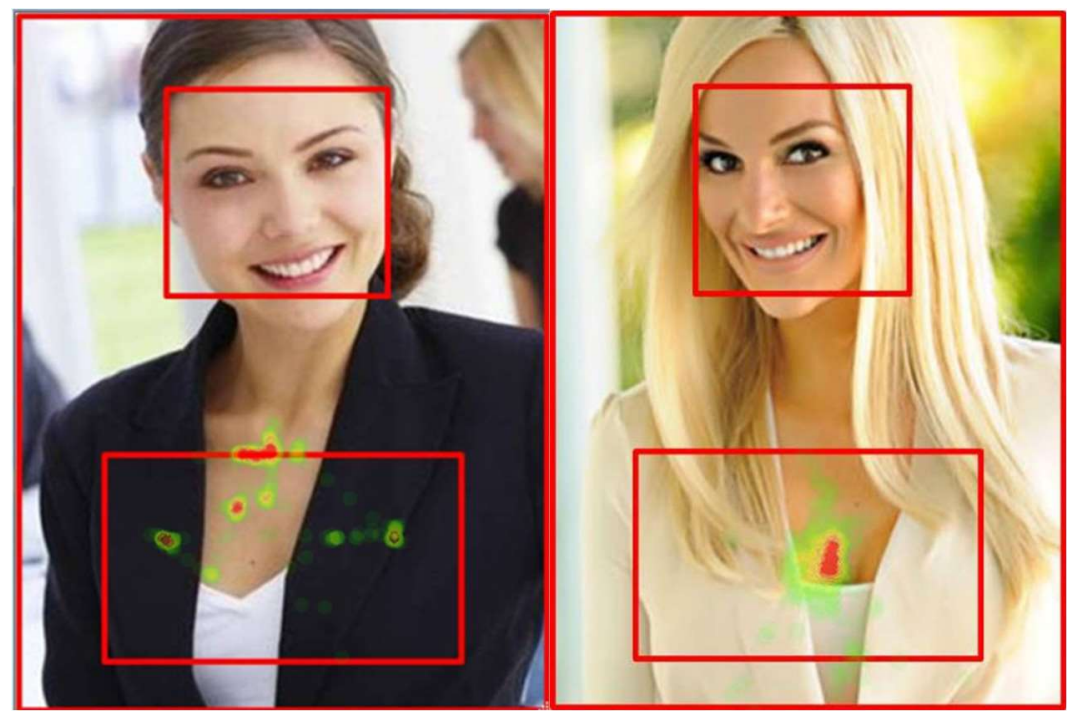

\section{Explicit and Implicit Outcomes}

Session $3(N=32)$ had the men from Session 2 interview a confederate female in a filmed interview. The male participant arrived and found the female confederate, posing as a fellow participant already waiting. The research assistant would flip a double-sided coin always assigning the male to the role of interviewer and the female confederate to the role of interviewed. The male was given a series of 5 questions and the female confederate always responded with the same generally positive responses. Afterward the men rated the interviewee (always the same scripted confederate) with a series of 5 Likert employment questions (explicit outcome: Cronbach's Alpha $=0.89$ ). The first measure of non-verbal behaviors was the distance between the interviewer and interviewee measured in 0-10 4-inch increments. (measured by a large wall map with time zones printed behind the interview area). Distances recorded ranged from 10 (maximum possible with secured chairs) to 5 closest possible while remaining seated). This measure was used to determine if the male interviewers were leaning in towards interview (showing interest) or away from interviewee (showing disinterest). A team of three research assistants rated each video (no sound) on the average distance and disagreements were discussed (none of the raters differed by more than 1 unit in their initial rating). The second 1-10 rating was provided by the confederate indicating the amount of eye contact being made by the interviewer. These two measures were highly correlated and were combined into a single implicit outcome score (Cronbach's Alpha = 0.73).

\section{RESULTS}

Hypothesis 1 was supported; the amount of time male participants fixed on the chests of pictures of women applicants (eye tracking) was significantly positively correlated with both hostile $(r(63)=.37, p=$ $.05)$ and benevolent $(r(63=.43, p=.03)$ sexism measured by the ASI. Hypothesis 2 and 3 were also supported. A model including explicit (benevolent and hostile sexism) and implicit measures (eye tracking) found that only eye tracking was a significant predictor of explicit candidate ratings and implicit non-verbal behaviors. Significant stepwise multiple regressions supported both hypotheses, (Model 1 explicit interview ratings: $R^{2}=.18 ; F(1,29)=6.42, p=.02$; Eye Tracking $B=.43, p=.02$ ), (Model 2 non-verbal behaviors; $R^{2}=.22 ; F(1,29)=8.29, p=.01$; Eye Tracking $\left.B=.47, p=.01\right)$. Greater time spent fixated on women's chests in the eye-tracking phase was the primary predictor of giving lower explicit ratings and less positive non-verbal behaviors (for example leaning in or away from the interviewee) when interviewing a female confederate. 


\section{DISCUSSION}

Men's increased fixations on women's chests as an indication of implicit bias was significantly indicative of being related to increased explicit sexism and predicted both explicit and implicit bias in interactions with a female interviewee. Limitations included the small student sample. The average time to calibrate and then track a single participant's eye fixations can be up to 30 minutes and only results in usable data about $80 \%$ of the time. Though, the initial session resulted in a large pool of potential applicants, only $39 \%$ continued to the eye tracking session and only $30 \%$ resulted in useable data, and of those, less than $50 \%$ continued into the final session. Future studies need to address issues of completion allowing for greater confidence and generalizability of these results.

Thousands of gender bias claims every year in the U. S. (EEOC) and the significant monetary, physical, and psychological toll to both organizations and individuals (Smith, et al., 2018) supports the necessity of continued research into further understanding sexism in the workplace. The present study illustrates the importance of understanding the connection between interviewers' sexist beliefs and interview outcomes for a female interviewee.

\section{REFERENCES}

Cornelissen, P.L., Hancock, P.J.B., Kiviniemi, V., George, H.R., \& Tovée, M.J. (2009). Patterns of eye movements when male and female observers judge female attractiveness, body fat and waist-tohip ratio. Evolution and Human Behavior, 30, 417-428.

Duchowski, A.T. (2002). A breadth-first survey of eye-tracking applications. Behavior Research Methods, Instruments, \& Computers, 34(4), 455-470.

Forbes, G.B., Collinsworth, L.L., Jobe, R.L., Braun, K.D., \& Wise, L.M. (2007). Sexism, hostility toward women, and endorsement of beauty ideals and practices: Are beauty ideals associated with oppressive beliefs? Sex Roles, 56, 265-273.

Gartner, R.E., \& Sterzing, P.R. (2016). Gender microaggressions as a gateway to sexual harassment and sexual assault: Expanding the conceptualization of youth sexual violence. Affilia, 31(4), 491-503

Gerpott, F., Lehmann-Willenbrock, N., Sivlis, J., \& Van Vugt, M. (2017). In the eye of the beholder? An eye-tracking experiment on emergent leadership in team interactions. The Leadership Quarterly. http://dx.doi.org/10.1016/j.leaqua.2017.11.003

Glick, P., \& Fiske, S.T. (2001). An ambivalent alliance: Hostile and benevolent sexism ascomplementary justifications for gender inequality. American Psychologist, 56(2), 109118.

Hayes, A., Pham, T., Phillips, L., \& Nadler, J. T. (2019, April). Eye tracking as a predictor of sexist attitudes and subsequent behaviors. In N. Hauensteins (Chair), Advances in measuring gender bias. Symposium presented at the 2019 annual meeting of the Society for Industrial and Organizational Psychology (SIOP). Washington, D.C.

Hewig, J., Trippe, R.H., Hecht, H., Straube, T., Miltner, W.H.R. (2008). Gender differences for specific body regions when looking at men and women. Journal of Nonverbal Behavior, 32, 67-78.

Nadler, J.T., \& Stockdale, M.S. (2012). Workplace Gender Bias: Not Between Just Strangers. North American Journal of Psychology, 14, 281-292

Smith, J., Liss, M., Erchull, M., Kelly, C., Adragna, K., \& Baines, K. (2018). The relationship between sexualized appearance and perceptions of women's competence and electability. Sex Roles. https://doi.org/10.1007/s11199-018-0898-4

Stockdale, M.S., \& Nadler, J.T. (2013). Paradigmatic assumptions to disciplinary research on gender disparities: The case of occupational sex segregation. Sex Roles, 68, 207-215.

Swami, V., \& Tovée, M.J. (2013). Men's oppressive beliefs predict their Brest size preferences in women. Archives of Sexual Behavior, pp. 1-9.

Zelazniewicz, A.M., \& Pawlowski, B. (2011). Female breast size attractiveness for men as a function of sociosexual orientation (restricted vs. unrestricted). Archives of Sexual Behavior, 40, 1129-1135. 